\section{Sugar coating bronchiectasis}

\author{
Michael Loebinger
}

Bronchiectasis is a condition characterised by damage and dilatation of airways. Clinically, this manifests with productive cough and recurrent infective exacerbations. The importance of bronchiectasis is increasingly becoming recognised with data demonstrating an increased prevalence and significant associated morbidity, mortality and healthcare burden. ${ }^{1}{ }^{2}$ This has stimulated a surge in interest from academics and industry alike, with the start of several multicentre clinical trials. These have however been limited in part by the heterogeneity of bronchiectasis, with multiple different causes and significant variability in disease progression. ${ }^{3}$ The development of bronchiectasis severity scoring systems ${ }^{4}$ has helped to stratify patients into prognostic groups; however, the underlying basis for much of this heterogeneity has not been determined.

In Thorax, Taylor et al investigate one possible genetic predisposition to a worse bronchiectasis phenotype. They used the well-published, prospective cohort from the BLESS trial, which assessed the impact of long-term erythromycin on exacerbation rate. ${ }^{6}$ They focus their attention on the ability of the 112 patients to secrete histo-blood group antigens which is known to be a genetically determined autosomal trait encoded by the FUT2 gene. ${ }^{7}$ This gene encodes fucosyltransferases, which act to attach fucose to disaccharide precursors and hence control the expression of $\mathrm{ABO}$ antigens on the epithelial mucin glycans ('secretors'). Approximately $25 \%$ of the population are known to be homozygous for nonsense mutations in the FUT2 gene which results in an inability to produce antigens on the epithelial surface and an absence of $\mathrm{ABO}$ antigens on secreted mucins, providing a different mucin glycan phenotype ('non-secretors'). ${ }^{7} \quad 8$ Microbes may use these carbohydrates for adherence, invasion, induction of virulence genes and as sources of carbon. ${ }^{8}$ Differences in such glycosylation between secretors and non-secretors may therefore impact the host-pathogen interaction, and previous studies have demonstrated that secretors are more susceptible to certain

Correspondence to Dr Michael Loebinger, Host Defence Unit, Division of Respiratory Medicine, Royal Brompton Hospital, London SW3 6NP, UK; M.Loebinger@rbht.nhs.uk pathogens such as Norovirus and respiratory viruses such as influenza, rhinovirus and respiratory syncytial virus, ${ }^{9}$ but less to others such as Candida albicans, Haemophilus influenzae, Neisseria meningitidis and Streptococcus pneumoniae. ${ }^{10}$

This study demonstrates that the secretors, with functional FUT2 genes, have a more severe bronchiectasis phenotype. The mean FEV1\% predicted at baseline was $61.6 \%$ in those with two functional alleles (SeSe) compared with $74.5 \%$ in those with a non-secretor (sese) genotype. Furthermore, secretors had significantly more exacerbations in the year prior to the trial (mean 5.77 vs 4.07 ) and in physician defined exacerbations during the prospective year of follow-up (mean 2.03 vs 1.15); however, differences in the time to next exacerbation were not statistically significant. Data are not provided on other measures of severity, in particular CT scanning and bronchiectasis severity scoring tools FACED or Bronchiectasis Severity Index which would have significantly improved the characterisation of these patients. ${ }^{4}$ Unlike other conditions, such as Crohn's disease, where secretor status has been demonstrated to confer risk of the disease, ${ }^{11}$ there was no increased secretor prevalence in the bronchiectasis cohort compared with population studies. This suggests that secretor phenotype was not an underlying cause or predisposing factor for bronchiectasis. This should however be examined further in a larger case-control study on an independent population. Secretor status may however affect the clinical course and severity of the established disease.

The underlying mechanism for the possible effects on bronchiectasis is unknown. The authors postulate that the differences in the mucin glycan phenotype provide the basis for an alteration of the microbial environment leading to increased infection and exacerbation and a worse clinical course. They assess the microbial environment with molecular microbiology techniques using $16 \mathrm{~S}$ rRNA sequencing rather than standard microbial culture, following a prior publication from the same group demonstrating association of data from this methodology with clinical outcomes (using the same group of patients as in this study). ${ }^{12}$ Secretors are shown to be more likely to have a 'Psendomonas sp. dominated' sample on sequencing and one hypothesis is therefore that the glycan differences lead to an increased susceptibility to Pseudomonas infection and a less favourable clinical course. However, as the authors acknowledge, the lack of Pseudomonas fuctose catabolism or adherence mechanisms specific for $(1,2)$ fucosylated glycan makes a direct link less likely. ${ }^{5}$ The alternative hypothesis that more subtle changes in the microbial community between secretors and nonsecretors could lead directly to a worse prognosis or via the increased susceptibility to Pseudomonas was also not supported by the lack of demonstrable difference in the microbiome analysis. This later finding is at odds with Crohn's disease whereby differences in secretor status have been shown to lead to alterations in the compositional and functional levels of the microbial community of the colon from molecular microbiology and metabolomics studies. ${ }^{13}{ }^{14}$ The final hypothesis is that viral infection leads to increased exacerbations, lower lung function and increased use of antibiotics that lead to increased susceptibility to Pseudomonas infection. There is no direct evidence for this proposal but weak support from the fact that secretors are more susceptible to viral infections ${ }^{10}$ and recent data demonstrating that viruses are more commonly detected by PCR in patients with bronchiectasis during an exacerbation than at stable state. ${ }^{15}$ Further studies would need to be performed directly assessing viral load during exacerbations.

The association between secretors and a more severe bronchiectasis phenotype is a plausible outcome as secretors have had a similar negative prognostic effect in patients with asthma, with secretors having a significantly higher risk of being a severe and regularly exacerbating patient with asthma in a case-control analysis. ${ }^{16}$ However, the situation in asthma is complicated by other studies suggesting that non-secretors are overrepresented and potentially more predisposed to asthma and wheeze in both adults and children. ${ }^{17}$ Furthermore, the assessment of the role of secretor status in other chronic respiratory disease muddies the water still further. There was no relationship between secretor status and severity or age of onset of chronic Pseudomonas infection in a retrospective study of 880 patients with cystic fibrosis (CF). ${ }^{18}$ A study in 1017 patients with COPD demonstrated the reverse association in comparison with this study, with non-secretors demonstrating more severe disease based on spirometry. ${ }^{19}$ It is unknown whether these differences point 
to an inconsistent effect or relate to the different underlying chronic pathologies, for example, COPD severity may be less driven by infection and changes in the microbial community, and changes in CF due to FUT2 status may be masked by the differing epithelial and mucus environment in these patients. However, viral infections have similarly been implicated as having a role in some exacerbations in these other chronic respiratory conditions. ${ }^{20}$

In any event, the data presented in this issue are from a small number of patients based on a single centre study with no external validation cohort. Furthermore, the inclusion criteria for patients in the original study selected a generally severe population, limiting the ability to demonstrate differences in severity of disease between the secretor types. Consequently, while these data are interesting, significant caution is required and the study would need to be repeated in different, independent bronchiectasis populations including a wider range of severities and potentially different bronchiectasis aetiologies.

There are few other studies that have looked at potential gene associations in bronchiectasis, with mannose binding lectin $^{21}$ and matrix metalloproteinase gene variants ${ }^{22}$ implicated in modifying bronchiectasis severity and conflicting reports on the association of Killer cell Immunoglobulin-like Receptors (KIR) and Human Leukocyte Antigen (HLA)-C type with susceptibility to bronchiectasis. ${ }^{23} 24$ Bronchiectasis is also included in the UK 100000 genome project where the whole genome sequencing approach may provide new genetic associations. This study is interesting and well performed and if it is a consistent and repeated finding in further studies, together with more evidence relating to the underlying mechanisms, then it may have implications for clinical management of patients with bronchiectasis. It does give some insight into the factors relating to pathogenesis and heterogeneity in bronchiectasis and may potentially help explain the differences in susceptibility to certain microbes. This in turn may provide the opportunity for more targeted monitoring strategies, particularly with regard to microbial status and may also lead to lower thresholds for therapies in certain patient groups. The potential alterations to the microbial community driven by these genetic changes may also provide different responses to present therapeutic options in bronchiectasis, in particular the use of long-term antibiotics. This study did not provide data on whether there was any difference in the response of the secretor and non-secretor groups to the long-term macrolide; however, numbers in these subgroups would have been low and not adequately powered to look at this.

There is much still to learn about bronchiectasis and the underlying heterogeneity, but understanding this further will be key to adequately designing and stratifying clinical trials and subsequently being able to better manage and assess patients appropriately. Recent studies have started to try and address this with phenotyping of patients by different methods such as by microbe, ${ }^{25}$ molecular microbiology, ${ }^{12}$ severity scoring system ${ }^{4}$ or clinical phenotype. $^{26}$ The goal of these methods, which is shared by this study, is to try and move closer to a precision medicine approach within bronchiectasis.

\section{Competing interests None declared.}

Provenance and peer review Not commissioned; externally peer reviewed.

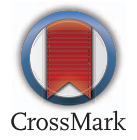

To cite Loebinger M. Thorax 2017;72:300-301.

Published Online First 9 January 2017

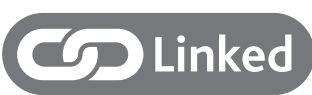

- http://dx.doi.org/10.1136/thoraxjnl-2016-208775

Thorax 2017;72:300-301.

doi:10.1136/thoraxjnl-2016-209274

\section{REFERENCES}

1 Quint JK, Millett ER, Joshi M, et al. Changes in the incidence, prevalence and mortality of bronchiectasis in the UK from 2004 to 2013: a population-based cohort study. Eur Respir J 2016;47:186-93.

2 Loebinger MR, Wells AU, Hansell DM, et al. Mortality in bronchiectasis: a long-term study assessing the factors influencing survival. Eur Respir J 2009;34:843-9.

3 Chalmers JD, Loebinger M, Aliberti S. Challenges in the development of new therapies for bronchiectasis. Expert Opin Pharmacother 2015;16:833-50.

4 Ellis HC, Cowman S, Fernandes M, et al. Predicting mortality in bronchiectasis using bronchiectasis severity index and FACED scores: a 19-year cohort study. Eur Respir J 2016;47:482-9.

5 Taylor SL, Woodman RJ, Chen AC, et al. FUT2 genotype influences lung function, exacerbation frequency and airway microbiota in non-CF bronchiectasis. Thorax Published Online First: 8 Aug 2016. doi: 10.1136/thoraxinl-2016-208775.

6 Serisier DJ, Martin ML, McGuckin MA, et al. Effect of long-term, low-dose erythromycin on pulmonary exacerbations among patients with non-cystic fibrosis bronchiectasis: the BLESS randomized controlled trial. JAMA 2013:309:1260-7.

7 Koda $Y$, Tachida H, Pang $\mathrm{H}$, et al. Contrasting patterns of polymorphisms at the $\mathrm{ABO}$-secretor gene
(FUT2) and plasma alpha(1,3)fucosyltransferase gene (FUT6) in human populations. Genetics 2001;158:747-56.

8 Ferrer-Admetlla A, Sikora M, Laayouni $H$, et al. A natural history of FUT2 polymorphism in humans. Mol Biol Evol 2009:26:1993-2003.

9 Raza MW, Blackwell CC, Molyneaux P, et al. Association between secretor status and respiratory viral illness. BMJ 1991;303:815-18.

10 Blackwell CC, Jónsdóttir K, Hanson M, et al. Non-secretion of $A B O$ antigens predisposing to infection by Neisseria meningitidis and Streptococcus pneumoniae. Lancet 1986;2:284-5.

11 McGovern DP, Jones MR, Taylor KD, et al. Fucosyltransferase 2 (FUT2) non-secretor status is associated with Crohn's disease. Hum Mol Genet 2010;19:3468-76.

12 Rogers GB, Zain NM, Bruce KD, et al. A novel microbiota stratification system predicts future exacerbations in bronchiectasis. Ann Am Thorac Soc 2014;11:496-503.

13 Tong M, McHardy I, Ruegger $\mathrm{P}$, et al. Reprograming of gut microbiome energy metabolism by the FUT2 Crohn's disease risk polymorphism. ISME J 2014;8:2193-206.

14 Rausch P, Rehman A, Künzel S, et al. Colonic mucosa-associated microbiota is influenced by an interaction of Crohn disease and FUT2 (Secretor) genotype. Proc Natl Acad Sci USA 2011;108:19030-5.

15 Gao YH, Guan WJ, Xu G, et al. The role of viral infection in pulmonary exacerbations of bronchiectasis in adults: a prospective study. Chest 2015; 147:1635-43.

16 Innes $\mathrm{AL}, \mathrm{McGrath} \mathrm{KW}$, Dougherty $\mathrm{RH}$, et al. The $\mathrm{H}$ antigen at epithelial surfaces is associated with susceptibility to asthma exacerbation. Am J Respir Crit Care Med 2011;183:189-94.

17 Kauffmann F, Frette C, Pham QT, et al. Associations of blood group-related antigens to FEV1, wheezing, and asthma. Am J Respir Crit Care Med 1996:153:76-82.

18 Taylor-Cousar JL, Zariwala MA, Burch LH, et al. Histo-blood group gene polymorphisms as potential genetic modifiers of infection and cystic fibrosis lung disease severity. PLoS ONE 2009;4:e4270.

19 Cohen BH, Bias WB, Chase GA, et al. Is ABH nonsecretor status a risk factor for obstructive lung disease? Am J Epidemiol 1980;111:285-91.

20 Hewitt R, Farne $H$, Ritchie $A$, et al. The role of viral infections in exacerbations of chronic obstructive pulmonary disease and asthma. Ther Adv Respir Dis 2016;10:158-74.

21 Chalmers JD, McHugh BJ, Doherty C, et al. Mannose-binding lectin deficiency and disease severity in non-cystic fibrosis bronchiectasis: a prospective study. Lancet Respir Med 2013;1:224-32.

22 Hsieh MH, Chou PC, Chou CL, et al. Matrix metalloproteinase-1 polymorphism (-1607G) and disease severity in non-cystic fibrosis bronchiectasis in Taiwan. PLOS ONE 2013;8:e66265.

23 Boyton RJ, Smith J, Ward R, et al. HLA-C and killer cell immunoglobulin-like receptor genes in idiopathic bronchiectasis. Am J Respir Crit Care Med 2006;173:327-33.

24 McDonnell MJ, Anwar GA, Rutherford RM, et al. Lack of association between KIR and HLA-C type and susceptibility to idiopathic bronchiectasis. Respir Med 2014; 108:1127-33.

25 Finch S, McDonnell MJ, Abo-Leyah $\mathrm{H}$, et al. A comprehensive analysis of the impact of pseudomonas aeruginosa colonization on prognosis in adult bronchiectasis. Ann Am Thorac Soc 2015;12:1602-11.

26 Aliberti S, Lonni S, Dore S, et al. Clinical phenotypes in adult patients with bronchiectasis. Eur Respir $J$ 2016:47:1113-22. 